\title{
High-Strain Fiber Bragg Gratings for Structural Fatigue Testing of Military Aircraft
}

\author{
Claire DAVIS*, Silvia TEJEDOR, Ivan GRABOVAC, \\ James KOPCZYK, and Travis NUYENS \\ Defence Science and Technology Organisation, 506 Lorimer St., Fisherman's Bend, VIC 3207, Australia \\ *Corresponding author: Claire DAVISＥ-mail: claire.davis@dsto.defence.gov.au
}

\begin{abstract}
This paper reports on an experimental program of work which investigates the reliability, durability, and packaging of fiber Bragg gratings (FBGs) for application as distributed strain sensors during structural fatigue testing of military platforms. The influence of the FBG fabrication process on sensor reliability is investigated. In addition, methodologies for broad-area packaging and surface-mounting of FBG sensing arrays to defense platforms are developed and tested.
\end{abstract}

Keywords: Fiber Bragg grating, draw tower grating, structural testing, strain sensor

\section{Introduction}

The structural airworthiness of the Royal Australian Air Force (RAAF) is founded on a rigorous program of full-scale fatigue testing. Test loads simulating the aircraft flight loading conditions are generated and applied to the aircraft using custom-designed servo-hydraulic or mechanical loading systems. These full-scale fatigue tests require detailed experimental strain data from across the structure to compare with strains measured on the flight test aircraft and to validate the strain fields predicted using fluid dynamics and finite element modeling under different flight conditions. In addition, the strain sensors are required to operate under relatively high strains for a large number of cycles in order to determine the fatigue limits of the structure. These measurements are normally made using conventional electrical resistance foil gauges. Each gauge requires two or three insulated leads which results in a complex sensing network as shown in Fig. 1. There is also significant weight associated with the foil gauge wiring which can affect the response of the structure. Furthermore, the foil gauges can sometimes be less durable than the structure under test which necessitates replacement of the foil gauges midway through the testing cycle.

Although fiber Bragg gratings (FBGs) offer obvious potential for use in these high-density, high-strain sensing applications, the adoption of this technology in the historically conservative aerospace industry has been slow. This can be attributed to many factors; the equipment required to interrogate the sensors is costly and often sensitive to harsh environments, there is a lack of conclusive information about sensor reliability and durability, and the existing electrical foil strain gauge technology is well-understood and suitable for most discrete sensing applications. In addition, there has been limited experience in the field with bonding and networking techniques for optical fiber based sensing networks particularly on large and/or complex structures.

Received: 6 June 2012 / Revised version: 10 June 2012

(C) The Author(s) 2012. This article is published with open access at Springerlink.com 


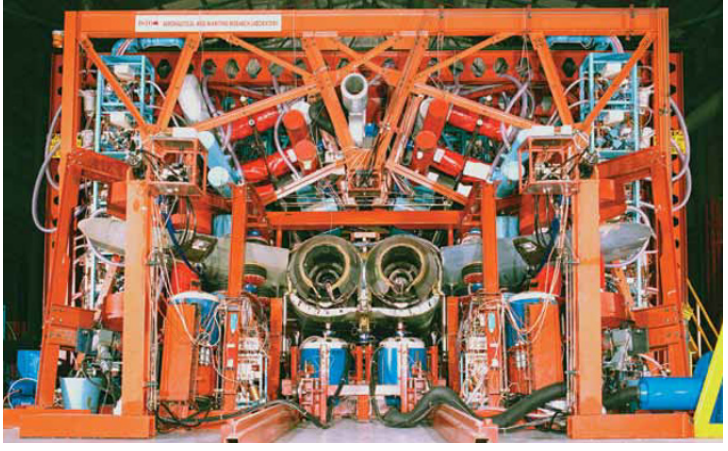

(a)

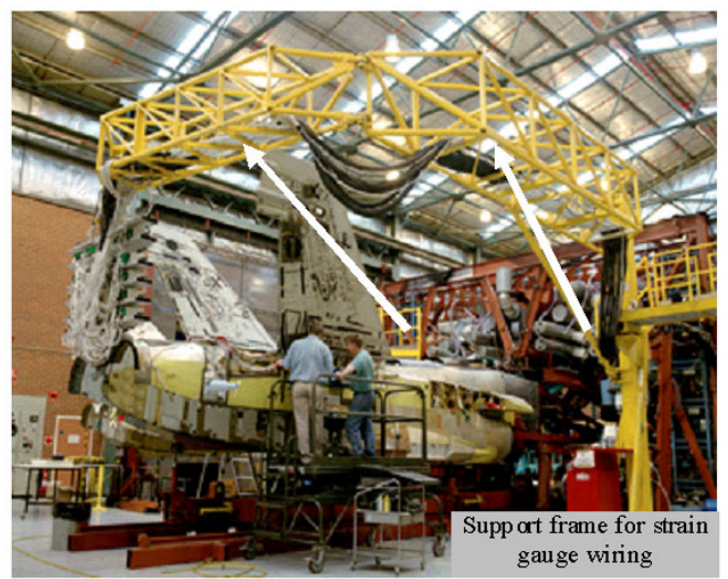

(b)

Fig. 1 Structural fatigue testing at the Defense Science and Technology Organisation (DSTO): (a) structural fatigue test of an F/A-18 aircraft and (b) inspection of the aft fuselage during an F/A-18 fatigue test (showing support frame to carry the strain gauge wiring).

Although optical fibers inherently offer excellent strength and resistance to environmental degradation due to the material properties of the glass used to make them, the reported reliability and durability of optical fibers containing FBGs have been variable, in part due to the fabrication methodologies used to create the sensors [1-3].

This paper studies the performance of FBGs inscribed during the fiber fabrication process known as draw tower gratings (DTGs) and compares them to the performance of FBGs which have been written in pre-fabricated optical fibers which have had their protective coating stripped in the region of the sensor. The performance of both types of the FBG is investigated under static tensile loading conditions on a series of fiber glass test-coupons using different adhesive packaging techniques custom-designed for the broad-area surface-mount application of high-density sensor arrays.

\section{FBG fabrication and packaging}

\subsection{Fabrication}

A Bragg grating is a periodic change in the refractive index in the core of an optical fiber. The periodic modulation is achieved by exposing the fiber (side-on) to an ultra violet (UV) laser beam. The region of the optical fiber exposed to the beam is altered via modification of the oxygen vacancy-defect absorption band resulting in a small increase in the refractive index. FBGs typically reflect light over a narrow wavelength range and transmit all other wavelengths. The relationship between the reflected wavelength $\lambda_{\text {Bragg }}$ and the period of the refractive index change can be approximated by (1):

$$
\lambda_{\text {Bragg }}=2 n_{\text {eff }} \Lambda
$$

where $n_{\text {eff }}$ is the effective refractive index of the fiber, and $\Lambda$ is the period of the grating. Changes in the axial strain and/or temperature in the region of the grating will alter the period of the index modulation and result in a shift in the reflected Bragg wavelength.

Because the hermetic coating on the optical fiber is usually not transmissive to UV light, there are typically four main steps involved in fabrication of a fiber Bragg grating:

1. Removal of the fiber coating.

2. Photosensitization of the fiber.

3. Exposure of the grating to UV laser light.

4. Annealing and re-coating.

Each one of these steps in the process has the potential to introduce structural flaws to the glass surface which weakens its ultimate strength and long-term reliability. For high density strain sensing applications where the coating is removed at many sections along the same fiber to inscribe multiple gratings, there is a much higher likelihood that the fiber will be damaged at some point along its length 
during the inscription process.

DTGs are Bragg gratings which are written into the optical fiber during the fiber fabrication process. Historically, these DTGs were characterized by very low reflectivities $(<1 \%)$ due to the fact that there was a limited time window available for UV exposure during the fiber draw process. In recent years with the advent of higher photosensitivity glasses and fiber draw-speeds which can be well-controlled over slower rates, DTGs have become available with reflectivities up to $30 \%-50 \%$ [4].

Figure 2 shows a schematic diagram of the production process for a DTG which involves a series of steps as summarized below:

1. The photo sensitive glass preform is heated up and drawn to initiate the formation of the fiber.

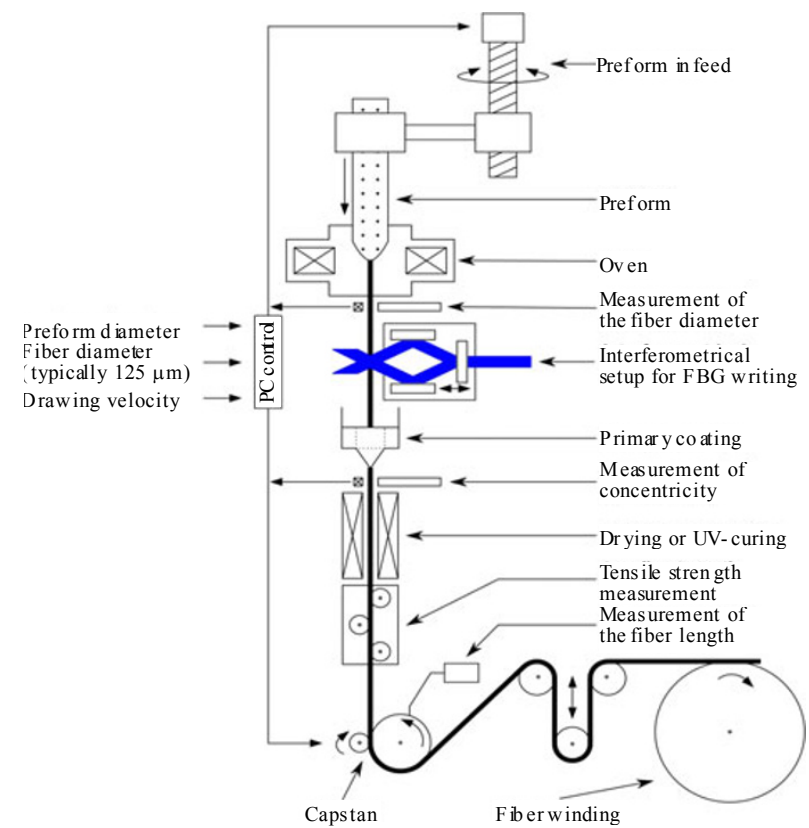

Fig. 2 Schematic representation of a draw tower setup (diagram supplied courtesy of FBGS International).

2. The drawn fiber crosses the optical axis of a pulsed excimer laser which uses an interferometric technique to expose the fiber with a periodic UV exposure pattern.

3. The fiber draw speed is monitored in combination with the laser pulse to control the exact location of the FBG sensors.
4. Once the grating has been written, the fiber is coated in the draw tower using a standard fiber coating process.

DTGs have been reported in the literature as exhibiting enhanced durability and increased strength because of the fact that the FBG is written immediately before the protective coating is applied to the fiber. This minimizes the potential for contamination of the exposed glass and avoids the requirement for harsh coating removal techniques [4].

\subsection{Packaging}

For broad-area structural assessment of large defense platforms, a robust and reliable technique is required for retro-fitting a high-density network of strain sensors onto a large structure. The packaging must not add significant weight or impact the surface profile of the structure under test. In addition, the material properties should ensure realistic strain transfer to the sensor(s), i.e. the use of the bonding medium must not contribute to significant shear lag, relaxation or creep under load. Generally, thermo-set resin systems are considered suitable as there is no measurable material distortion after full cure. For large-scale applications where hundreds of sensors may be used, a methodology for handling and placement must be developed. Therefore from a practical viewpoint, the pre-packaging should involve some form of shielding to protect fibers during handling and transportation as well as a methodology for accurately positioning the location of each sensor.

With these considerations in mind, two different packaging techniques were developed at DSTO to facilitate surface-mounting of high sensor count FBG arrays to defense platforms. The first technique is a vacuum assisted resin transfer molding (VARTM) process. VARTM is a closed moulding technique where a vacuum pressure draws the resin along a distribution medium. It is typically a three-step process including the lay-up of reinforcing fiber preform, preform impregnation 
with resin, and ambient or elevated temperature resin cure.

For small scale infusions, the first layer of the VARTM preform is a pressure sensitive, self-adhesive tape which is used for placement, transportation, and temporary adhesion of the fiber optic network. The second layer is a permeable peel ply which is used to separate the resin distribution media from the adhesive after resin cure. The third layer of the VARTM lay-up acts as the resin distribution medium. The resin is a low-viscosity thermoset system designed to achieve good wetting and high-strength, low-creep adhesion [5].

For large scale infusions, the VARTM lay-up incorporates multiple resin injection ports. Figure 3 shows the laboratory trials for a 3-meter infusion with three embedded optical fibers.

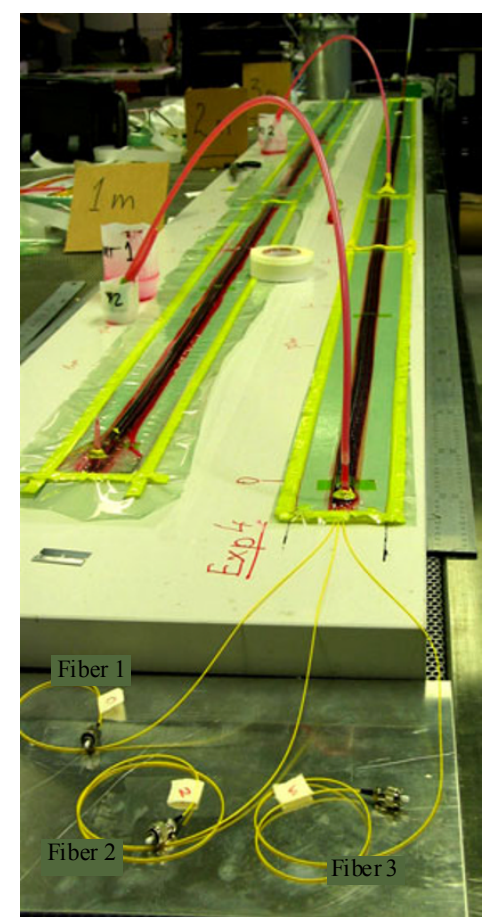

Fig. 3 Large-scale VARTM infusion trial with multiple resin injection ports.

Figure 4 shows the detail from the lay-up, infusion, and post-cure stages of the VARTM process.

The second packaging technique developed utilizes a nylon woven carrier tape impregnated with a modified epoxy film adhesive (Redux $\left.{ }^{\circledR}\right)$. This material was originally designed for maintaining uniform bond line thickness in composite bonding for aerospace applications. The optical fibers are firstly laid up under tension beneath one or more plies of the film adhesive tape in a mould which is vacuum sealed as shown schematically in Fig. 5(a). The number of plies of the film adhesive can be adjusted to suit the thickness and flexibility required for the tape. A single ply will result in a final tape thickness of approximately 250 microns while two plies will result in a thickness of approximately 440 microns. The tape is then cured under vacuum at an elevated temperature which depends on the variant of the Redux ${ }^{\circledR}$ film used $\left(120^{\circ} \mathrm{C}\right.$ for $\operatorname{Redux}{ }^{\circledR} 312,175{ }^{\circ} \mathrm{C}$ for Redux $\left.{ }^{\circledR} 322\right)$. This tape can either be co-cured with the structure or cured separately with the optical fiber, as shown in Fig. 6, and then secondarily bonded to the structure.

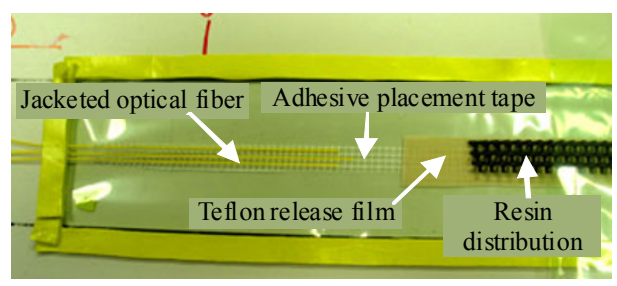

(a)

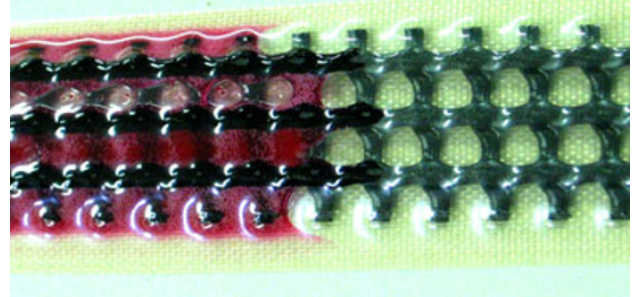

(b)

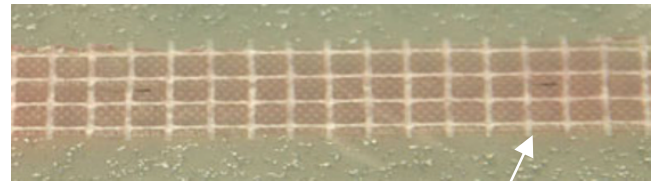

(c)

Fig. 4 Close-up photographs showing the VARTM process: (a) VARTM lay-up detail under vacuum seal, (b) close-up of the advancing resin front (dyed to aid viewing), and (c) close-up section of the completed VARTM package (after removal of sacrificial layers). 


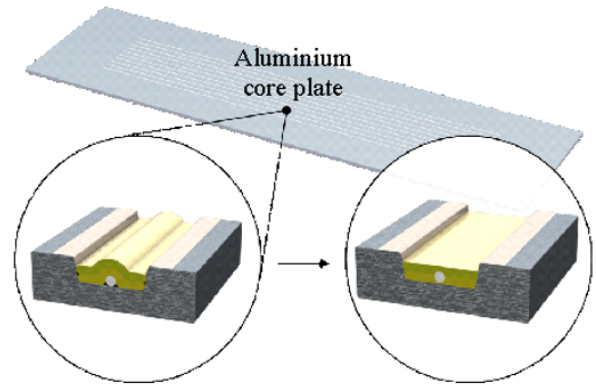

(a)

(b)

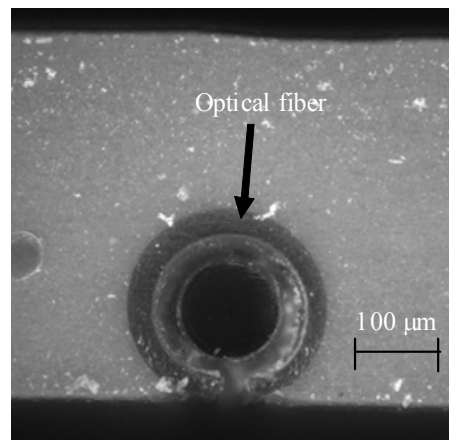

(c)

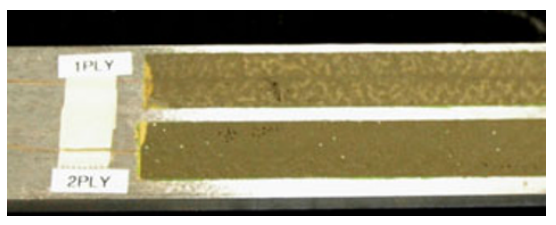

(d)

Fig. 5 Schematic diagram showing Redux ${ }^{\circledR}$ tape lay-up with the embedded optical fiber: (a) prior to cure, (b) post cure, (c) the microscopic image showing the cross section of 2-ply Redux ${ }^{\circledR}$ tape, and (d) single and two-ply Redux ${ }^{\circledR}$ tape with embedded optical fibers shown cured directly to the aluminium sample.

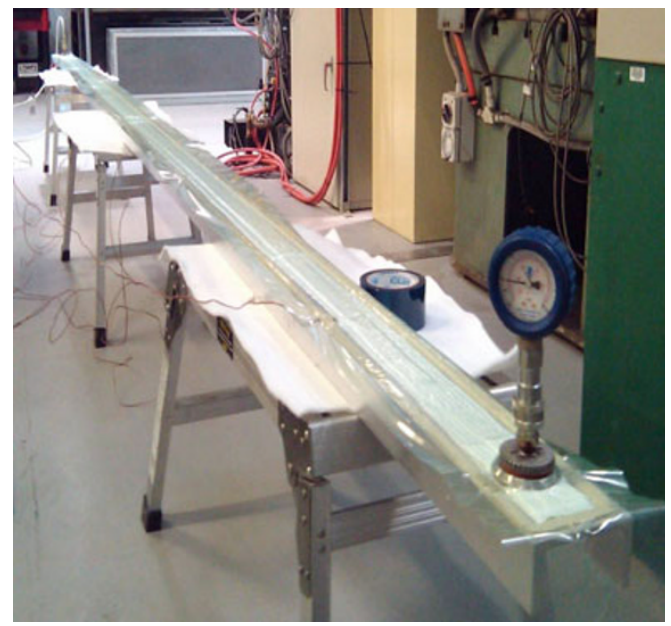

Fig. 6 Photograph showing 5.5-m length of Redux ${ }^{\circledR}$ tape under vacuum bag, being cured using a thermal blanket.
Both packaging techniques have been trialed for broad area surface-mounting of FBG arrays. The VARTM technique is applied at room temperature, and the infused packaging has a final thickness of $1 \mathrm{~mm}-3 \mathrm{~mm}$ depending on the resin distribution medium. The $\operatorname{Redux}{ }^{\circledR}$ tape has a lower impact on the surface profile of the structure $(250 \mu \mathrm{m}-600 \mu \mathrm{m})$ but can only be applied to materials or structures which can withstand temperatures of at least $120{ }^{\circ} \mathrm{C}$ if co-cured directly to the structure.

\section{Experimental methodology}

The reliability and durability testing for the FBGs and their packaging was conducted on high-strain test coupons in three stages. In the first stage tests, the strain-to-failure of FBGs inscribed into pre-fabricated optical fibers was investigated. For these FBGs, the protective coating on the optical fiber was removed using a selection of the most common coating removal techniques prior to inscription of the grating.

A custom-designed mounting assembly was developed to house the stripped fibers during hydrogenation and prior to inscription of the FBG to avoid any accidental physical contact of the exposed glass surface. The stripped fibers were hydrogenated for five days to photosensitize the fiber prior to grating inscription using a standard phase mask exposure technique [6]. All the fibers were stored in a desiccator cabinet post-inscription and prior to application to the test coupon to minimize moisture ingress to the exposed glass and were packaged within 48 hours of inscription.

The second stage of testing compared the performance of the best performing FBGs from the first stage testing to commercially supplied DTGs.

In the third stage of testing, the DTGs were dynamically loaded in tension with a sinusoidal load resulting in peak strains up to $10000 \mu \varepsilon$.

Two types of glass fiber reinforced composite (GFRC) coupons were fabricated for the testing program using MTM57 E-glass pre-preg. The first 
set of coupons comprised 12 unidirectional plies $[0]_{12}$ with dimensions of $200 \mathrm{~mm} \times 25 \mathrm{~mm}$ manufactured to American Society for Testing and Materials (ASTM) standards (D3039). The second set of coupons comprised 12 plies laid up in a cross-ply orientation $\left[(+45,-45)_{3}\right]_{\mathrm{s}}$ with dimensions of $200 \mathrm{~mm} \times 25 \mathrm{~mm}$ also manufactured to ASTM standards. Aluminium alloy grip tabs were bonded to either side of the top and bottom of each coupon to protect the coupon from the load-cell grips during tensile loading. The uni-directional test coupons had a predicted strain-to-failure of $20000 \mu \varepsilon$ with a linear stress-strain response for most of the strain range to failure. The cross-ply coupons were designed to test the ultimate strength of the optical fibers. These coupons had a much higher strain-to-failure, with a low material yield point followed by a significant region of non-linear plastic deformation.

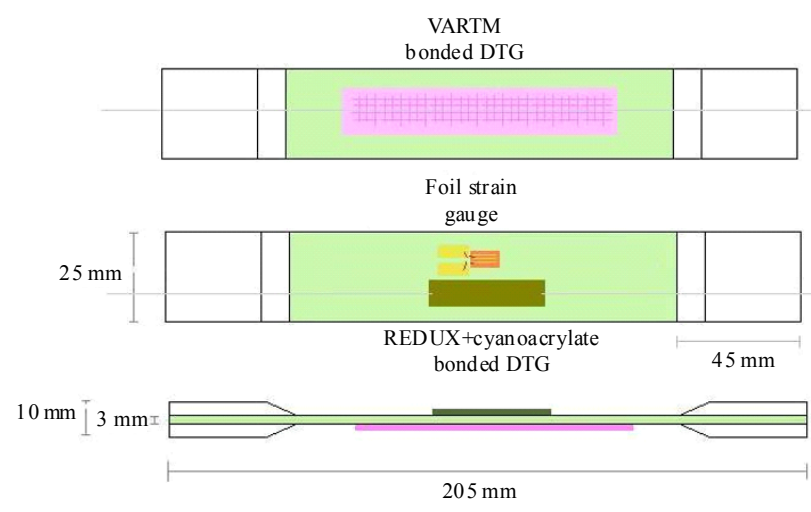

Fig. 7 Top, bottom, and side views of the GFRC coupon showing placement of packaged and bonded sensors.

The VARTM technique provides both packaging and direct bonding of the DTG sensor to the test coupon. However, it was not possible to cure the Redux ${ }^{\circledR}$ tape directly to the test coupons as the E-glass material could not withstand the Redux ${ }^{\circledR}$ cure temperature. Thus, pre-cured Redux ${ }^{\circledR}$ tape sections containing the embedded FBGs were secondarily bonded to the surface of the test coupons using a standard strain gauge adhesive (micro-measurements M-Bond 200). M-Bond 200 is a rapid curing cyano-acrylate adhesive which is designed to produce a creep-free, fatigue-resistant bond with an elongation capability of $5 \%$. Figure 7 shows a schematic diagram of a typical test coupon showing the location and orientation of the FBGs and their packaging. A conventional electrical resistance foil gauge was also bonded to the test coupon to provide an independent measure of strain for the strain-to-failure tests.

\subsection{Stage 1 testing}

The first stage testing was designed to investigate the effect of different coating removal techniques on the ultimate tensile strength of the optical fibers. It involved measuring the strain-to-failure of a series of optical fibers which had their coating removed using five different methods as outlined below:

1. Thermal wire stripper

A shaped heating element $\left(900^{\circ} \mathrm{C}\right)$ removes the coating thermally.

2. Butane flame

A low temperature/high oxygen mix butane flame (cigarette lighter) burns the PI coating, leaving a carbon residue which requires cleaning using iso-propyl alcohol and lint-free wipes.

3. Butane torch

A high temperature butane flame from a pressurised canister thermally ablates the PI coating.

4. $\mathrm{CO}_{2}$ laser

Laser ablation of the acrylate coating is achieved using a commercially supplied $\mathrm{CO}_{2}$ laser stripping system from $\mathrm{Oz}$ Optics. A $100-\mathrm{W} \mathrm{CO}_{2}$ laser beam at $9.3 \mu \mathrm{m}$ is focused on the fiber to ablate the coating.

5. Heated sulphuric acid

Immersion in the concentrated (99.9\%) sulphuric acid at $120^{\circ} \mathrm{C}$ for 20 minutes to chemically remove the coating.

After removal of the fiber coating and hydrogenation, an FBG was inscribed in the stripped section with a continuous wave frequency-doubled argon ion laser using a standard phasemask exposure technique [6]. In total, 30 FBGs were tested using 
the five different coating removal techniques. The FBGs were either surface mounted using the VARTM or Redux packaging techniques or embedded between plies 6 and 7 of the unidirectional GFRC test coupons.

The unidirectional test coupons were loaded in tension using a $100-\mathrm{kN}$ material test system (MTS) at $1-\mathrm{kN}$ interval, and the reflection spectrum from each FBG was recorded for each load using an optical spectrum analyzer; the load was increased until optical fiber failure.

\subsection{Stage 2 testing}

The second stage of testing compared the performance of the best performing FBGs from the first stage testing to commercially supplied DTGs acquired from FBGS International.

The lay-up of the composite test coupon was altered from unidirectional to a cross-ply configuration to achieve a higher strain range (up to $80000 \mu \varepsilon)$. A total of 26 cross-ply coupons were prepared for this stage of testing. A single FBG or DTG was surface-mounted front and back to each coupon using the VARTM and Redux packaging techniques, respectively as shown in Fig. 7.

Static loads were applied at $1-\mathrm{kN}$ interval from $0 \mathrm{kN}$ to $4 \mathrm{kN}$. From $4 \mathrm{kN}$, a ramp load was applied at a rate of $500 \mathrm{~N} / \mathrm{min}$ until failure of the sensors. Each FBG/DTG was interrogated using an optical spectrum analyzer via an optical fiber switch box, and the reflection spectrum for each grating was recorded automatically every twenty (20) seconds until failure of the sensors.

\subsection{Stage 3 testing}

The final stage of testing investigated the fatigue performance of the draw tower gratings in both the Redux and VARTM packaging. A total of 26 unidirectional coupons were fabricated for this stage of the testing. The coupons were sinusoidally loaded in tension to induce a peak strain level in the coupon of $10000 \mu \varepsilon$. The frequency of the loading was limited to $6 \mathrm{~Hz}$ to prevent heating in the specimen.
Two DTGs were applied to each coupon using the VARTM and Redux ${ }^{\circledR}$ packaging techniques, respectively as represented schematically in Fig. 7. A foil resistance strain gauge was not used for strain measurement on these coupons due to the poor fatigue performance of the foil gauges at these strain levels.

The dynamic strain response of the DTGs during the cyclic loading was measured using a peak-tracking commercial Bragg grating interrogation system (Smart Fibers Wx). The reflection spectrum of each DTG was also measured at regular interval during the testing regime, using a static strain survey to determine whether there was any deterioration in the reflection spectrum as cycling progressed.

\section{Results}

Figure 8 shows the average strain-to-failure of the stripped FBGs plotted by the coating removal technique. The results indicate that there is a large degree of variation in strain-to-failure for each coating removal method and that the overall strain-to-failure is significantly lower than the predicted strain-to-failure for a pristine optical fiber which is stated to be about $5 \%$ or $50000 \mu \varepsilon$. Overall, the $\mathrm{CO}_{2}$ laser ablated fibers demonstrated the highest strain-to-failure of all the coating removal techniques, and this coating removal method was used for the second stage tests.

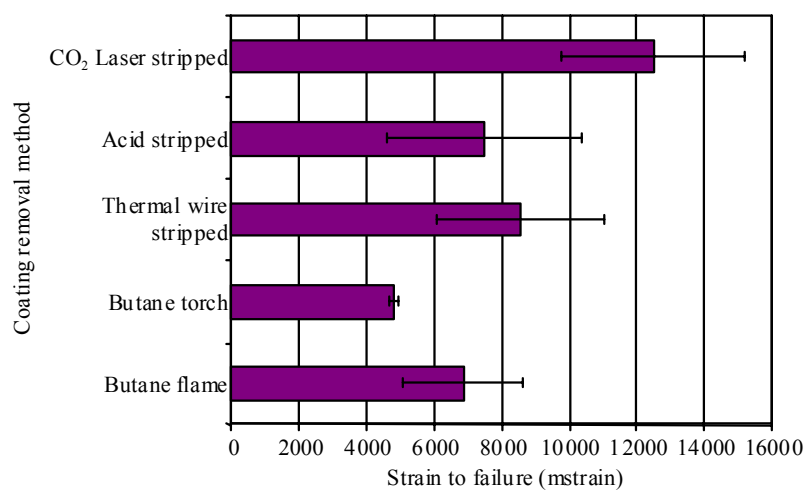

Fig. 8 Average strain-to-failure for different coating removal techniques (error bars indicate standard deviation of results for each measurement group). 
Figure 9 compares the average strain-to-failure levels for the $\mathrm{CO}_{2}$ stripped FBGs with the DTGs for both the VARTM and Redux ${ }^{\circledR}$ packaging. The results indicate that for both packaging techniques, the strain-to-failure for the DTGs is approaching the expected level for that of the pristine optical fiber (approximately $5 \%$ or $50000 \mu \varepsilon$ ) whereas the average strain-to-failure levels for the FBGs in the $\mathrm{CO}_{2}$ laser stripped fibers are significantly lower than that for the DTGs.

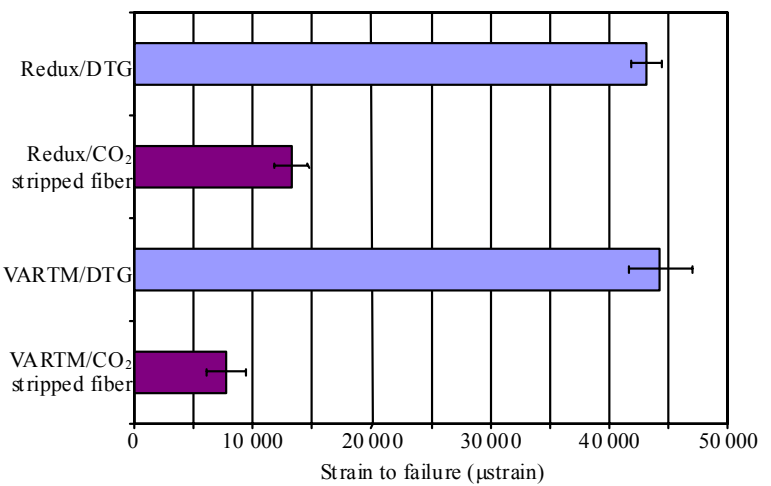

Fig. 9 Comparison of the average strain-to-failure for DTGs and $\mathrm{CO}_{2}$ laser stripped FBGs in Redux ${ }^{\circledR}$ and VARTM packaging.

Figure 10 shows the fatigue life (no. of cycles) for the DTGs subjected to the tensile cyclic loading to 10 $000 \mu \varepsilon$.

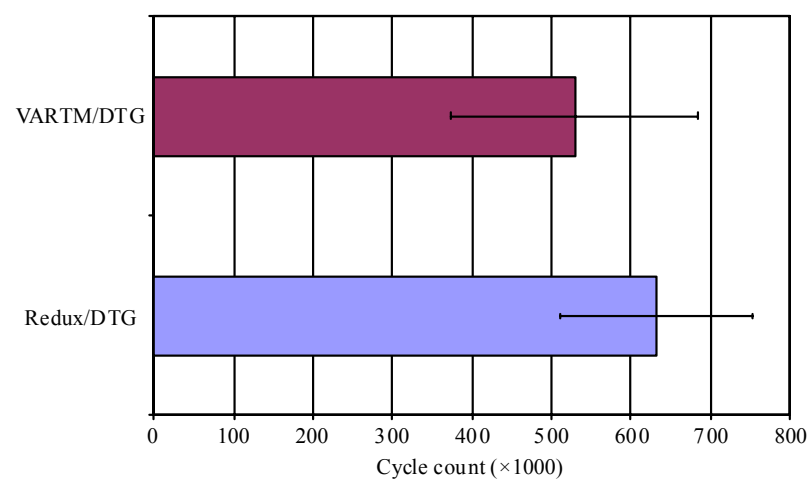

Fig 10 Comparison of the average fatigue life for DTGs in Redux ${ }^{\circledR}$ and VARTM packaging when subject to the cyclic sinusoidal loading with a peak strain of $10000 \mu \varepsilon$.

The results indicate that the Redux ${ }^{\circledR}$ packaging performs slightly better than the VARTM packaging under cyclic loading conditions although there is a large standard deviation for both data sets. As the cyclic loading progressed beyond 300 thousand cycles, there was also evidence of strain gradients along the length of the grating as indicated by peak splitting of the FBG reflection spectra under load, particularly for the VARTM packaging as shown in Fig. 11.
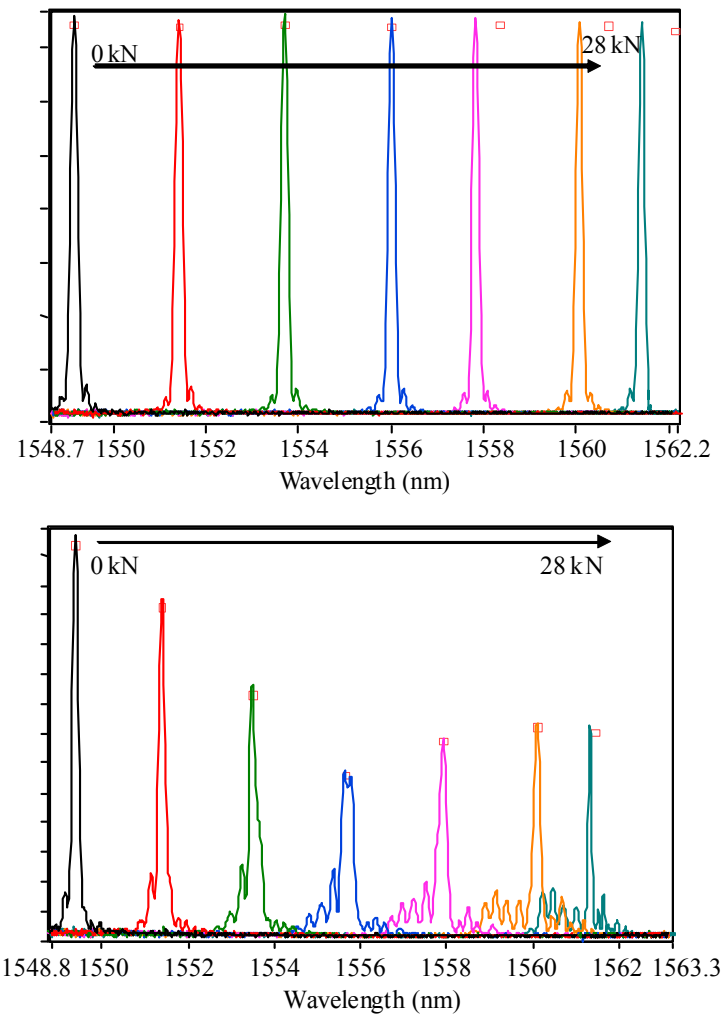

Fig. 11 Reflection spectra from static strain survey of the surface-mounted (VARTM) FBG up to $10000 \mu \varepsilon$ (top) prior to cycling (bottom) after $300 \mathrm{k}$ cycles.

The broader non-uniform spectral profiles with the reduced peak intensity are typically an indication of non-uniform strain profiles being experienced by the FBGs over the length of the gauge. A microscopic inspection of the packaging was conducted in order to verify whether micro-cracking of the resin matrix might be occurring prior to failure of the optical fiber as shown in Figs. 12 and 13.

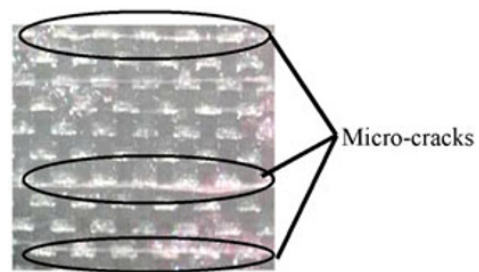

Fig. 12 Microscopic image showing micro-cracking in VARTM packaging after fatigue loading. 
Both packaging techniques show evidence of micro-cracking initiating after approximately 300000 cycles.

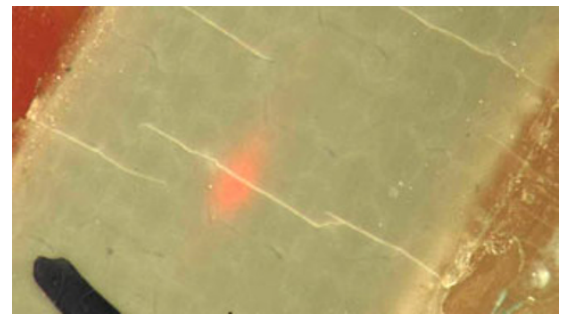

Fig. 13 Micro-crackings in Redux ${ }^{\circledR}$ packaging.

\section{Conclusions}

The results of the investigation into the effect of the manufacturing process on the reliability and durability of fiber Bragg gratings clearly showed that DTGs demonstrated significantly better performance than FBGs which were written into stripped and re-coated fibers.

With recent enhancements in the DTG fabrication technology, the reflectivity of these gratings should be suitable for use with most commercially available FBG interrogators which employ peak-tracking algorithms. There are however some challenges associated with the fabrication process used to write DTGs which limit the reflectivity, gauge length, and sensor density achievable for this class of gratings.

In situations where draw tower gratings are not suitable for the application, careful consideration should be given to the fabrication process employed by the manufacturer and the performance specifications supplied for these FBG sensors. The results from this investigation show that there is a large degree of variability in the performance of stripped and re-coated gratings which is influenced by both the coating removal technique and the handling, storage process during fabrication. This is probably a contributing factor to the large range of results which were reported in the literature in the area of FBG reliability.
Two broad area packaging techniques were developed to retrofit FBG/DTG sensing arrays to structural fatigue testing of the military aircraft. The preferred packaging technique largely depends on the targeted platform, the intended position of the sensors, the sensor count, and the conditions under which the sensors are expected to operate. Although both of the broad-area packaging techniques developed under this research program showed evidence of micro-cracking of the resin matrix under fatigue loading, the strain levels tested were well in excess of what would typically be experienced on most military platforms or applied during full-scale fatigue testing. In general, the laboratory trials to date have indicated that VARTM is more suited to the application on rigid structures with simple geometry where the sensor arrays can be rolled out onto a structure and then infused without any additional heating. Redux is more suited to high density sensing in less accessible locations or on more complex geometry as the Redux ${ }^{\circledR}$ tape has greater ductility and a smaller footprint.

\section{Acknowledgement}

The authors gratefully acknowledge the Office of Naval Research for the support of this work under the Naval International Cooperative Opportunities in Science and Technology Program (Grant No. N00014-09-1-0364; program sponsor Dr. Ignacio Perez). The authors would also like to acknowledge Mr. Peter Lombardo and Mr. Chris Hamill for assistance with the composites fabrication and Swinburne University for providing access to the $\mathrm{CO}_{2}$ laser for laser ablation of the fiber coatings.

Open Access: This article is distributed under the terms of the Creative Commons Attribution License which permits any use, distribution, and reproduction in any medium, provided the original author(s) and source are credited. 


\section{References}

[1] J. Schijve, "Fatigue of aircraft materials and structures," International Journal of Fatigue, vol. 16, no. 1, pp. 21-32, 1994.

[2] U. G. Goranson, "Fatigue issues in aircraft maintenance and repairs," International Journal of Fatigue, vol. 19, no. 93, pp. 3-21, 1997.

[3] L. Molent and S. A. Barter, "A comparison of crack growth behaviour in several full-scale airframe fatigue tests," International Journal of Fatigue, vol. 29, no. 6, pp. 1090-1099, 2007.

[4] H. J. Yoon and C. G. Kim, "The mechanical strength of fiber Bragg gratings under controlled UV laser conditions," Smart Materials Structures, vol. 16, no. 4, pp. 1315-1319, 2007.

[5] D. H. Kang, S. O. Park, C. S. Hong, and C. G. Kim, "Mechanical strength characteristics of fiber Bragg gratings considering fabrication process and reflectivity," Journal of Intelligent Material Systems and Structures, vol. 18, no. 4, pp. 303-309, 2007.

[6] A. J. Tarpey, S. N. Kukureka, and K. Jurkschat, "The mechanical reliability of stripped and recoated polyimide fibers for optical fiber sensors," in Proc. SPIE (Optical Fiber and Fiber Component Mechanical Reliability and Testing II), vol. 4639, pp 141-151, 2002.

[7] J. Ang, H. C. H. Li, and I. Herszberg "Tensile fatigue properties of fiber Bragg grating optical fiber sensors," International Journal of Fatigue, vol. 32, no.
4, pp 762-768, 2010.

[8] M. Rothhardt, C. Chojetzki, and H. R. Mueller, "High mechanical strength single-pulse draw tower gratings," in Proc. SPIE(North 2004: Photonic Applications in Telecommunications, Sensors, Software, and Lasers), vol. 5579, pp.127-135.

[9] C. Chojetzki, M. Rothhardt, J. Ommer, S. Unger, K. Schuster, and H. R. Mueller, "High-reflectivity draw-tower fiber Bragg gratings arrays and single gratings of type II," Optical Engineering, vol. 44, no. 6, pp. 060503, 2005.

[10] C. Y. Wei, C. C. Ye, S. W. James, R. P. Tatam, and P. E. Irving, "The influence of hydrogen loading and the fabrication process on the mechanical strength of optical fibre Bragg gratings," Optical Materials, vol. 20, no. 4, pp. 241-251, 2002.

[11] I. Grabovac, T. Nuyens, and C. Davis, "Packaging and mounting of in-fiber Bragg grating arrays for SHM of large structures," DSTO Technical Report, DSTO-TR-2490, 2010.

[12] A. Rizk and C. Davis "Reliability and durability studies for fabricating, packaging and bonding fibre Bragg gratings," in Proceedings Optical Fiber Technology (ACOFT), $2010 \quad 35$ th Australian Conference on optical fibre technology (IEEE 10.1109/ACOFT.2010.5929878), Dec. 5-9, pp. 1-4, 2010.

[13] Y. J. Rao, "In-fiber Bragg grating sensors," Measurement Science and Technology, vol. 8, no. 4, pp. 355-375, 1997. 